\title{
High Order APSK Constellation Design for Next Generation Satellite Communication
}

\author{
Farbod Kayhan*
}

\begin{abstract}
Employing high order constellations is inevitable in order to achieve high spectral efficiency in satellite communication systems. In DVB-S2X standard, constellations with up to 256 points have been included. However, optimizing such high order constellations is a difficult task. In this paper, we study circular constellations obtained as the image of the QAM under the radial map. Constellations with 16, 64 and 256 points are shown to have a mutual information very close to the peak power limited capacity for a large range of SNR values. For constellations with 256 points gains of at least $0.5 \mathrm{~dB}$ can be obtained with respect to the DVB-S2X standard. To have a more realistic view, we also consider the effect of the phase noise on our proposed constellations.
\end{abstract}

\section{Nomenclature}

AMI average mutual information, $I($.

APSK amplitude and phase-shift keying

AWGN additive white Gaussian noise

BICM bit interleaved coded modulation

DVB digital video broadcasting

HPA high power amplifier

LDPC low density parity check

MODCOD modulation and coding

OBO output back-off

PAMI pragmatic average mutual information, $I_{p}($.

PNSD phase noise standard deviation

PSNR peak signal power to noise ratio

QAM quadrature amplitude modulation

QCI QAM to circular isomorphic

SNR signal to noise ratio

\section{Introduction}

In view of the growing demand for spectral efficiency in satellite communications, high order modulation design has received a considerable attention in the past few years. Modulation and coding configurations employing constellations of orders 64,128 and 256 are adopted in the new digital video broadcasting standard (DVB-S2X). ${ }^{1}$ Even larger constellations may be needed in near future given the fact that the traffic demand for satellite broadband is expected to grow six-fold by $2020{ }^{2}$

Due to the presence of the HPA on board of the satellites, the conventional additive white Gaussian noise (AWGN) channel with average power limitation is no longer an accurate model for satellite communication systems and nonlinear characteristics have to be taken into account. When the HPA operates near the saturation point, its effects can be approximated by assuming a peak power limited signaling. The capacity achieving distribution, in this case, is proved to be discrete in amplitude (having finite number of mass points) with a uniformly distributed phase. ${ }^{3}$ The optimal distribution for a finite constellation is not known in general case. However, previous studies indicate that amplitude and phase-shift keying (APSK) modulations perform very close to the peak power limited capacity. ${ }^{4}$

\footnotetext{
* Research associate at Interdisciplinary Centre for Security, Reliability and Trust (SnT), university of Luxembourg, L-2721, Luxembourg. Email: farbod.kayhan@uni.lu
} 
Optimizing APSK constellations has been considered by several authors previously. The number of points on each circle, the radii of the rings and the phases of points on each ring need to be optimized in order to achieve near capacity performances. ${ }^{5}$ For constellations with up to 64 points, the number of possibilities is rather limited and therefore the optimization problem can be handled using gradient descent or exhaustive algorithms. For larger constellations the problem becomes complex and the proposed algorithms in the literature usually fail to provide a good sub-optimal solution. Another problem which rises regarding the optimization of APSK constellations is the bit to symbol mapping or the so called labelling of the constellation points.

Recently, a simple construction for non-uniform APSK constellations has been proposed by the author. ${ }^{6}$ The proposed constellations are the image of $M$-QAM under the radial map and are referred to as QCI constellations. In the same paper, it is shown that gains up to $1.5 \mathrm{~dB}$ can be obtained with respect to the constellations employed in DVB-S2X standard for constellations with 256 points. In this paper, we first provide a short review of the results presented in ${ }^{6}$ and then study various aspects of QCI constellations. We also extend the results to 16 and 64 point constellations. In particular, we show that for $M$-QCI constellations with $M=16,64$ and 256, the AMI is very close to the capacity limit for a wide range of SNRs. For even values of $m=\log _{2} M$, number of points on concentric circles of QCI constellations form an arithmetic progression with common difference 8 and starting point 4. For example, the number of points over 256-QCI rings are respectively $4,12,20,28,36,44,52$ and 60 . High number of points over one ring usually results in a bad performance over the channels with strong phase noise. In this paper we also evaluate the AMI in the presence of phase noise under the peak power constraint for QCI constellations.

The rest of this paper is organized as follows. In Section II we describe the notations and the objective functions upon which the constellations are compared. We also describe the QCI construction in details. In Section III, we first present the AMI curves for the QCI constellations and then compare them with those of DVB-S2X. In section IV we study the effect of the phase noise on QCI constellations. We discuss some of the ongoing and future research directions in Section V. Finally, we conclude the paper in Section VI.

\section{Notations and background}

Recently, a family of non-uniform APSK constellation has been proposed in ${ }^{6}$ by the author. The idea is to map a $M$-QAM constellation to a circular constellation through the radial map $f: \mathbb{R}^{2} \rightarrow \mathbb{R}^{2}$ given by:

$$
f(x, y)=\left\{\begin{aligned}
\frac{\sqrt{2} \max (|x|,|y|)}{\sqrt{x^{2}+y^{2}}}(x, y) & \text { if }(x, y) \neq(0,0) \\
(0,0) & \text { if }(x, y)=(0,0)
\end{aligned}\right.
$$

As examples, the images of 64-QAM and 256-QAM constellations under the radial map are shown in figures 1 (a) and 1(b), respectively. As it can be seen, the resulting constellations are non-uniform APSK constellations where the number of points on rings form an arithmetic progression with common difference 8 and starting point 4. Notice that the binary Gray labelling of QAM constellation is preserved under $f$, and the resulting QCI constellations have also a Gray labelling. Therefore, no extra labelling design is needed for QCI constellations. For constellations with 32 and 128 points, we consider the crossed shaped QAM constellations which are used commonly in the literature.

AMI has been shown to be a reliable metric for measuring the performance of a constellation over a given channel. ${ }^{4}$ For the AWGN channel, the received signal can be written as $\mathbf{y}=\mathbf{x}+\mathbf{n}$, where $\mathbf{n}$ is the additive white noise with Gaussian distribution. We denote the AMI of a given constellation $\chi$ by $I(\chi)$ :

$$
I(\chi)=\frac{1}{M} \sum_{\mathbf{x} \in \chi} E_{\mathbf{n}}\left\{\log \frac{P(\mathbf{y} \mid \mathbf{x})}{P(\mathbf{y})}\right\}
$$

where $E_{\mathbf{n}}$ is the average with respect to the Gaussian noise and $I(.,$.$) is the mutual information function.$ To calculate $E_{\mathbf{n}}$, we use a Gaussian quadrature approximation method.

As mentioned, we are interested in the peak-power limited design and therefore in what follows we always fix the maximum power of a constellation to be 1 , i.e., $|\mathbf{x}|^{2} \leq 1$ for all $\mathbf{x} \in \chi$. In such cases, instead of the signal to noise ratio, the peak power to noise ratio (PSNR) should be considered as the measure for comparing the performance of two constellations. Notice that PSNR is the ratio between the constellation's power and 


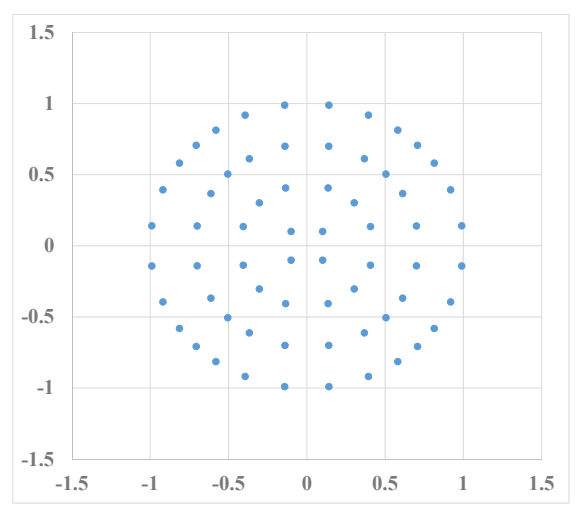

(a) 64-QCI.

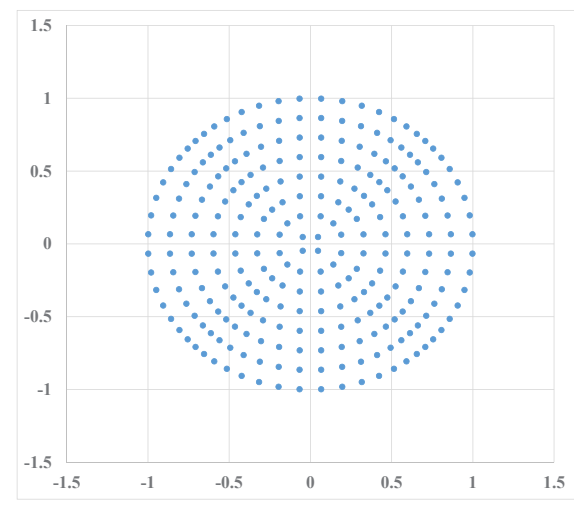

(b) 256-QCI.

Figure 1. 64-QCI and 256-QCI constellations obtained as the image of the 64-QAM and 256-QAM constellations under the radial map.

the noise power spectral density:

$$
\mathrm{PSNR} \triangleq \frac{1}{N_{0}} \geq \mathrm{SNR} \triangleq \frac{1}{M} \sum_{\mathbf{x} \in \chi} \frac{|\mathbf{x}|^{2}}{N_{0}}
$$

Assuming a memoryless ideal non-linearity model (soft limiter) for the HPA and ignoring the effect of filters, the PSNR coincides with $P_{\text {sat }} / N_{0}$ :

$$
\mathrm{SNR}=\frac{E_{s}}{N_{0}}=\frac{P_{\mathrm{sat}}}{N_{0}} \cdot \frac{E_{s}}{P_{\mathrm{sat}}}=\mathrm{PSNR}-O B O[\mathrm{~dB}] .
$$

\section{AMI computation for QCI constellations and comparison with DVB-S2X}

In this section we first compute the AMI for $M$-QCI constellations and then compare the results with those in DVB-S2X. In figure 2 we plot the AMI curves for the $M$-QCI constellations with $\mathrm{M}=16,32,64$, 128 and 256. We also plot the peak power limited capacity ${ }^{3}$ (also called Shamai capacity). Notice that the AMI loss from the capacity for $M$-QCI constellations is very small (around $0.1 \mathrm{~dB}$ ) for PSNR ranges of interest.

Similar to the $M$-QAM constellations, there is a degradation of performance for $M$-QCI constellations for odd values of $m$, i.e., for $M=32$ and $M=128$. We replot the AMI results only for $\mathrm{M}=16,64$ and 256 in figure 3. As it can be seen, by choosing low code rates with 256-QCI and high code rates with 64-QCI constellation, one may indeed avoid using the 128 point constellations with a very small loss. The same observation is also valid for the constellations with 32 points. To see this fact better, we also plot the loss from the capacity of $M$-QCI constellations in figure 4 . The red line indicates the loss of 0.1 bits/symbol from the peak power capacity. This figure indicates that no gain can be obtained by using 128-QCI constellation if the code rates are selected carefully for MODCODs with 64-QCI and 256-QCI constellations. Notice that the 128-QCI is obtained through the radial map of the crossed shaped 128-QAM constellation. For the 32-QCI constellation, the gains which can be obtained are limited to a small range of PNSRs. It is important to notice that $M$-QCI constellations are not optimal and in particular, 32 and 128 point constellations with higher AMI values can be easily found (see the Appendix for an example).

The DVB-S2X standard implements various constellations with 256 points (3 of which are APSK), but to the best of the author's knowledge, no justification about the constellation design has been provided. ${ }^{1}$ For the sake of completeness, we report again the AMI comparison results presented in ${ }^{6}$ between 256 -QCI and the 256 point constellations of DVB-S2X. Those readers familiar with the results presented in ${ }^{6}$ may skip the rest of this section. 


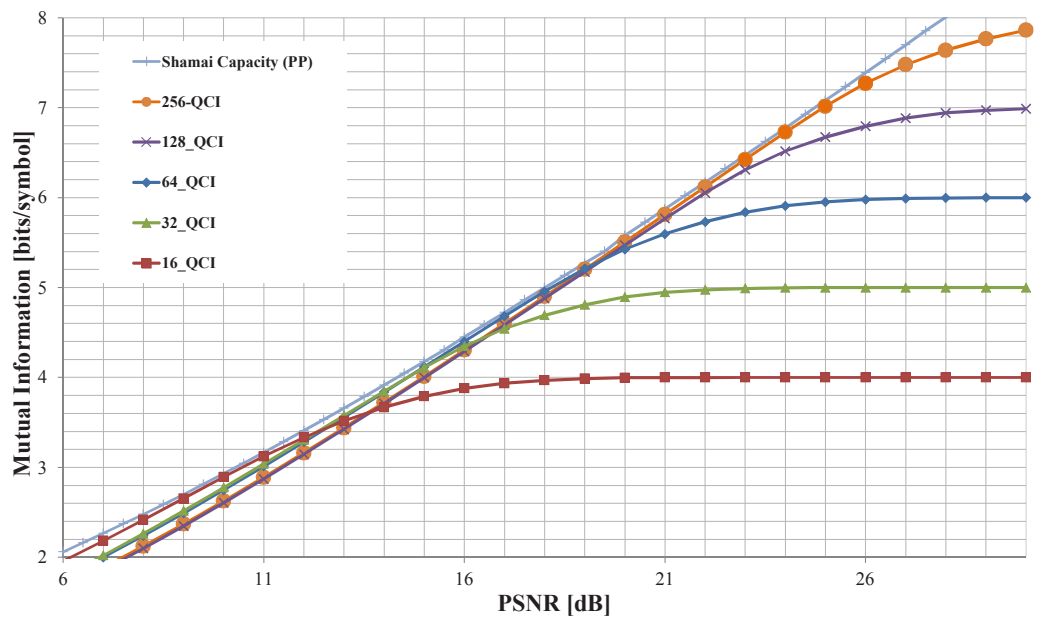

Figure 2. AMI curves as a function of PNSR for $M$-QCI constelations with $M=16,32,64,128$ and 256.

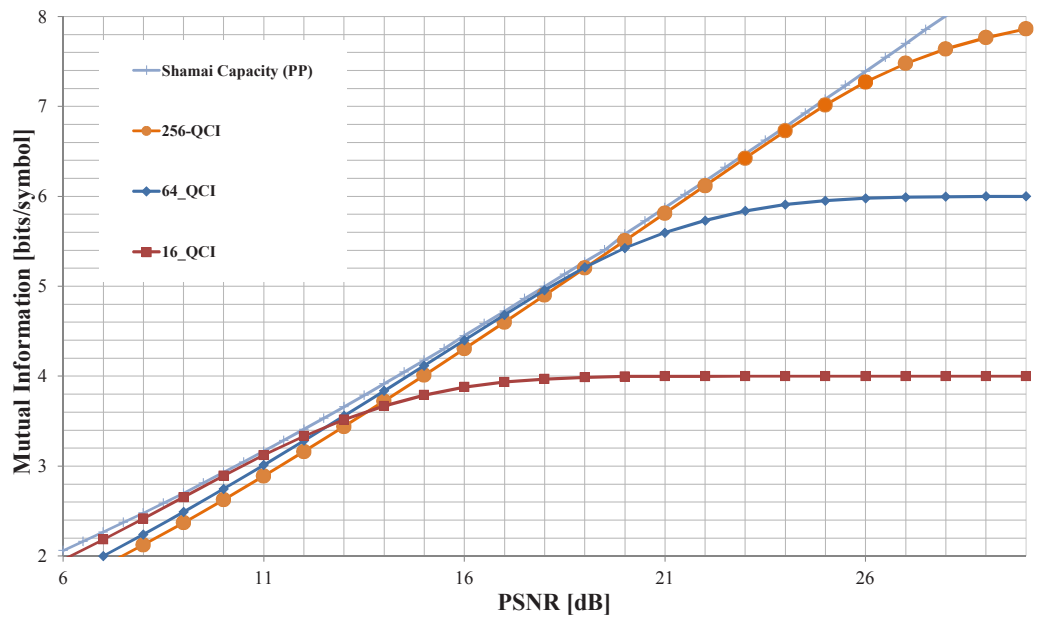

Figure 3. AMI curves as a function of PNSR for $M$-QCI constelations with $M=16,64$ and 256.

Five different constellations with 256 points are introduced in DVB-S2X standard. ${ }^{1}$ We distinguish these five constellations by the LDPC code rates they are coupled with in the DVB-S2X MODCODs. For example, the constellation coupled with the LDPC with rate 135/180 is denoted by "DVBS2X 135/180".

In figure 5 we compare the AMI of the 256-QCI and all five constellations of DVB-S2X. At one bit lower than the saturation point, QCI shows a gain of $0.7 \mathrm{~dB}$ with respect to the best constellation in DVBS2X. Targeting lower spectral efficiencies, the gain reduces to $0.5 \mathrm{~dB}$. We also plot the peak-power-limited capacity $^{3}$ in the same figure for comparison.

\section{Performance over the phase noise channel}

The 256-QCI constellation has 60 points over its outer ring. Such a high number of points over a single ring may degrade the performance of this constellation over the channels with phase noise. In this section we investigate the effect of the phase noise on the performance of 256-QCI constellation. Following the techniques introduced in ${ }^{12}$ we compute the AMI for the AWGN channel under the peak power constraint assuming a memoryless phase noise modeled as Tikhonov distribution.

In figure 6 we show the AMI curves for 256-QCI and two of the DVB-S2X constellations as a function 


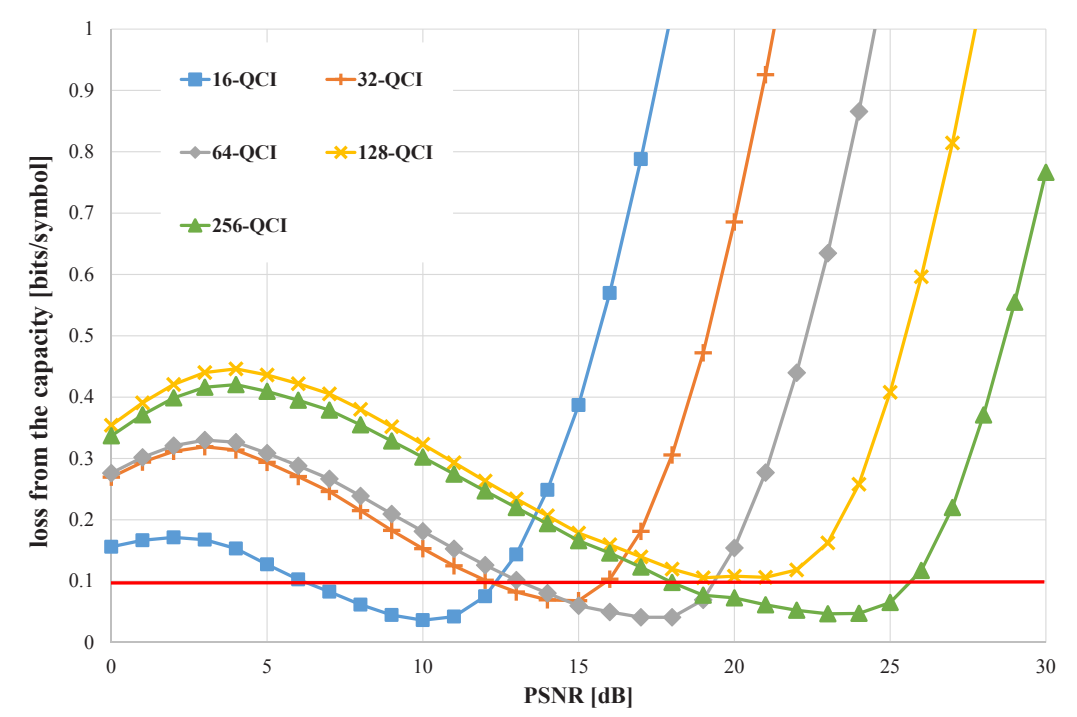

Figure 4. Loss from the peak power limited capacity for $M$-QCI constellations.

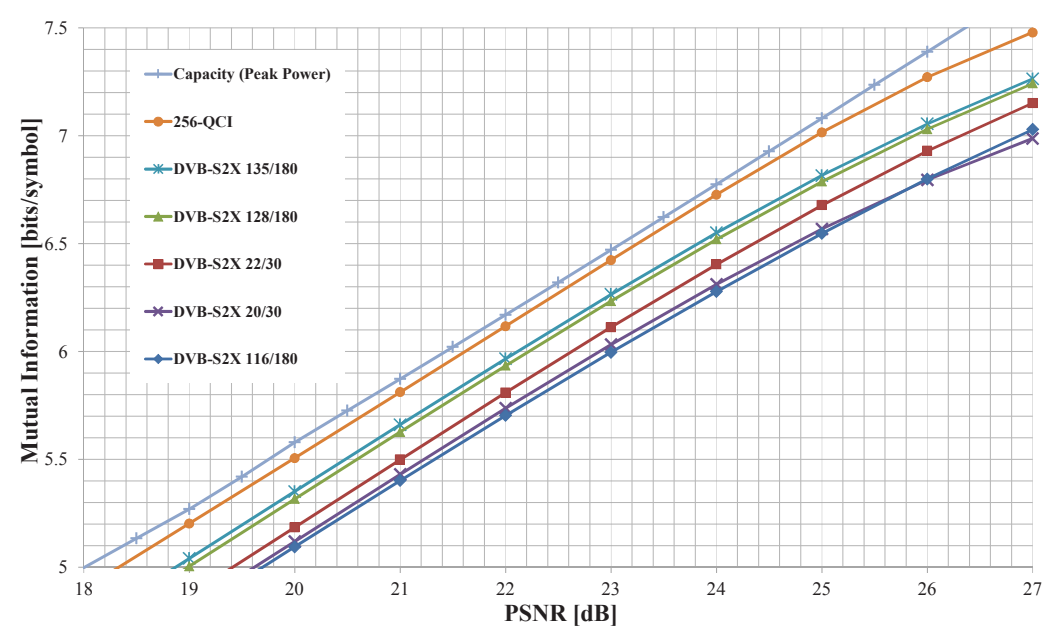

Figure 5. AMI comparison between 256-QCI and all DVB-S2X constellations.

of PNSD. The PSNR is fixed to $22 \mathrm{~dB}$ in all cases. The 256-QCI has a larger AMI in comparison to "DVB-S2X 135/180" constellation for PNSD up to 7 degrees. The residual phase noise standard deviation of the professional phase noise mask used in the DVB-S2X standard is around 2.5 degrees. For such values of PNSD, 256-QCI outperforms all the DVB-S2X constellations. The gains vary from $0.3 \mathrm{~dB}$ up to $1 \mathrm{~dB}$ depending on the MODCOD.

\section{Future research}

In this paper we only present the AMI results for the QCI constellations. Even though AMI is a reliable measure for performance estimation, end-to-end simulations are still needed to ensure that the promised gains can be achieved in a complete chain. In particular, in DVB-S2X standard, one needs to carefully choose the labelling of the constellations to match the optimized LDPC code through the interleaver.

Another direction for further research is to investigate the low complexity detection strategies for the QCI constellations. The simplest strategy is to apply the inverse map $f^{-1}$ at the receiver assuming that the signals originally have been chosen from a QAM constellation. In this way, one can use the low complexity 


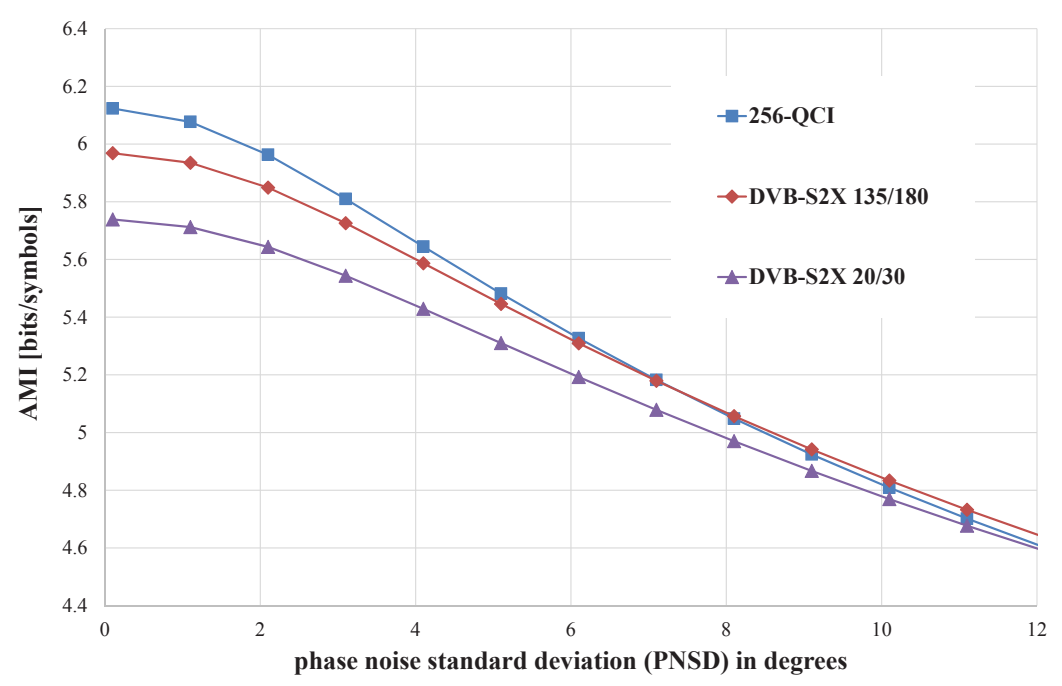

Figure 6. AMI curves for a AWGN channel with memoryless phase noise modeled as the Tikhonov ditricuted.

detection for QAM, based on the fact that it can be written as the Cartesian product of two one dimensional constellations. Our preliminary results show that this strategy will result in a loss of around $0.8 \mathrm{~dB}$. The main reason is that after applying $f^{-1}$ to the received signal $\mathbf{y}$, the noise is not any more Gaussian and the noise variance will also change. Therefore, more elaborate computation of the $P(\mathbf{y} \mid \mathbf{x})$ may be needed at the receiver. Techniques similar to those in ${ }^{14}$ may be also investigated in order to reduce the detection complexity.

Finally, it is important to notice that the QCI constellations are not optimal. The AMI for 16, 64 and 256 point QCIs are very close to the peak power capacity. However, the BICM capacity (also referred to as PAMI in the literature) may be improved. This can be done, for example, by optimizing the radii of the concentric rings of the QCI constellation (see also the appendix).

\section{Conclusion}

In this paper we study the $M$-QCI constellations obtained by the radial projection of $M$-QAM. First we show that the AMI of $M$-QCI constellations are very close (around $0.1 \mathrm{~dB}$ ) to the peak power capacity for the SNR ranges of interest. In particular, we show that by limiting the constellation's orders to 16,64 and 256 and choosing carefully the code rates, constellations with 32 and 128 points may be avoided with a small loss. Moreover, we show that by employing 256-QCI constellations, gains up to 1.5 dB may be possible with respect to the current DVB-S2X standard. Further, we also investigate the performance of 256-QCI constellations over the phase noise channels.

\section{Appendix}

As we have mentioned before, 32-QCI and 128-QCI constellations have a larger loss from the capacity in comparison to 16, 64 and 256 QCI constellations. As it can be observed in figure 4, if a loss of 0.1 bits/symbol is permitted, one may avoid using constellations with 32 and 128 points. For example, considering the 128QCI case, any spectral efficiency which can be achieved by this constellation can also be achieved using 64-QCI or 256-QCI constellation with a carefully chosen rate without any further loss. This is though due to the fact the 32-QCI and 128-QCI are not optimal constellations.

One can indeed optimize the constellations with 32 and 128 points under the peak power constraint. We have used the simulated annealing algorithm (initializing by QAM constellation and imposing a symmetry condition) to optimize these constellations. The resulting constellations are shown in figures 7(a) and 7(b) respectively. We refer to these constellation as 32-OPT and 128-OPT. We plot the loss from the capacity of these constellations in figure 8. As it can be noticed, rather significant gains with respect to the 32-QCI 


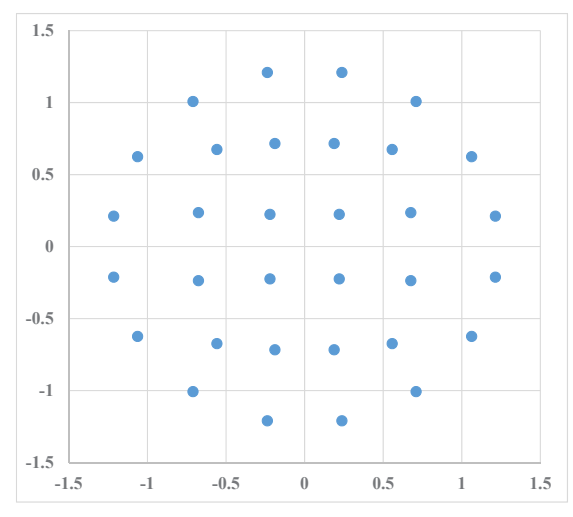

(a) 32-OPT.

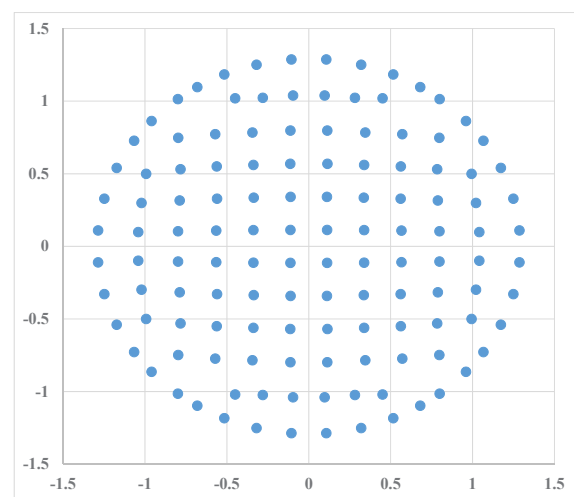

(b) $128-\mathrm{OPT}$.

Figure 7. 32 and 128 points optimized constellations under the peak power constraint using the simulated annealing algorithm.

and 128-QCI can be obtained. Our results indicate that even over the phase noise channels the 256-QCI constellation outperforms the DVB-S2X constellations.

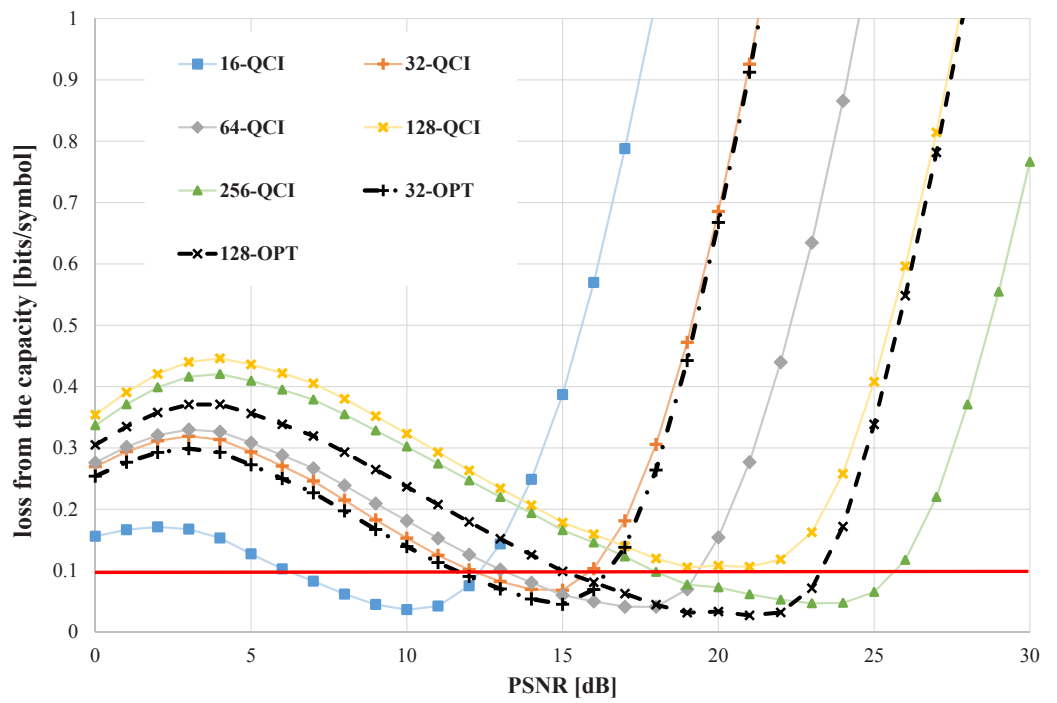

Figure 8. Loss from the capacity for 32-OPT and 128-OPT constellations and comparision with QCI constellations.

\section{Acknowledgments}

The author would like to thank Alberto Mengali, Bhavani Shankar and Bjorn Ottersten for some inspiring discussions.

This activity is partially supported by the European Space Agency (ESA) under the TRP contract "Optimized Transmission Techniques for SATCOM Unicast Interactive Traffic,", Contract no. ITT A0/1$8332 / 15 / \mathrm{NL} / \mathrm{FE}$. The views of the authors do no reflect the views of ESA. 


\section{References}

${ }^{1}$ ETSI 302-307-2, "Digital Video Broadcasting (DVB) Part II: DVBS2-Extensions (DVB-S2X)," 2014.

2 "Broadband Access via Integrated Terrestrial and Satellite Systems (BATS). D4.1: Satellite Network Mission Requirements," Technical Report, European Project, Avanti PLLC, 2012.

${ }^{3}$ S. Shamai (Shitz) and I. Bar-David, "The capacity of average and peak-power-limited quadrature Gaussian channels," IEEE Transaction on Information Theory, vol. 41, pp. 1061-1071, July 1995.

${ }^{4}$ F. Kayhan and G. Montorsi, "Joint signal-labeling optimization under peak power constraint," Int. J. of Satellite Communications and Networking, DOI: 10.1002/sat.1016, 2012.

${ }^{5}$ R. De Gaudenzi, A. Guillen i Fabrigas and A. Martinez, "Performance analysis of turbo-coded APSK modulations over nonlinear satellite channels," IEEE Trans. Wireless Communications, vol. 5, pp. 23962407, 2006.

${ }^{6} \mathrm{~F}$. Kayhan, "QAM to circular isomorphic constellations", $8^{\text {th }}$ Advanced Satellite Multimedia Systems Conference (ASMS), palma de mallorca, Sept. 2016.

${ }^{7} \mathrm{H}$. Meric, "Approaching the Gaussian Channel Capacity With APSK Constellations" in IEEE Communications Letters, vol. 19, no. 7, pp. 1125-1128, July 2015 .

${ }^{8}$ G. Caire, G. Taricco, and E. Biglieri, "Bit-interleaved coded modulation," IEEE Transaction on Information Theory, vol. 44, pp. 927-946, May 1998.

${ }^{9}$ F. Kayhan and G. Montorsi, "Constellation design for transmission over non-linear satellite channels," in IEEE Glob. Telecommun. Conf. (GLOBECOM), Annaheim, USA, Dec. 2012.

${ }^{10}$ A. J. Kearsley, "Global and local optimization algorithms for optimal signal set design," J. Res. Nat. Inst. Stand. Technol., vol. 106, pp. 441-454, 2001. 1983.

${ }^{11}$ C. D. G. S. Kirkpatrick and M. P. Vecchi, "Optimization by simulated annealing," Science, vol. 220, pp. 671-680, May

${ }^{12}$ F. Kayhan and G. Montorsi, "Constellation design for memoryless phase noise channels",IEEE Transactions on Wireless Communications, vol.13, no.5, pp.2874,2883, May 2014.

${ }^{13}$ G. Montorsi and F. Kayhan, "Analog Digital Belief Propagation and its Application to Multi-Stage Decoding Systems," 2015 IEEE International Black Sea Conference on Communications and Networking (BlackSeaCom), pp.82-86, May 2015.

${ }^{14}$ K. Kim, N. Basutkar, K. Bae, P. Xue and H. Yang, "One-Dimensional Soft-Demapping Algorithms for Rotated QAM and Software Implementation on DSP," IEEE Transactions on Signal Processing, vol. 61, no. 15, pp. 3918-3930, Aug.1, 2013. 